\title{
Exoskeleton Gait Training with Traditional Physical Therapy Management of a Patient with Polytrauma Injuries and Diffuse Axonal Injury: A Case Report
}

\author{
Bethany Spain, Man-Soo Ko \\ University of Texas Medical Branch
}

\begin{abstract}
Purpose People with polytrauma injuries along with diffuse axonal brain injury (TBI+DAI) present unique challenges to the physical therapist during comprehensive inpatient rehabilitation. The literature available to rehabilitation professionals regarding exoskeleton gait training (EGT) in the traumatic brain injury (TBI) population is limited. The purpose of this case report is to describe the use of EGT and traditional physical therapy management of an adult with polytrauma injuries and TBI+DAI during an inpatient rehabilitation stay. Methods The subject was a 31 year old veteran male diagnosed with polytrauma injuries and TBI+DAI, resulting from a high speed motor vehicle collision. He began to emerge from comatose state 8 days post-injury, was admitted to an inpatient rehabilitation facility and began physical therapy 23 days post-injury. He participated in physical therapy one to two times daily for 60 minute sessions with five additional EGT sessions and two community re-integration outings, for an average of 7 sessions per week throughout episode of care. Results Measures were taken at admission and were reassessed weekly. Functional Independence Measure total score increased from 62 to 117 and the motor subscale increased from 37 to 89 . Berg Balance Scale score increased from 0 to 54. Gait distance increased from 20 feet with quad cane and moderate assistance from physical therapist to 5,755 feet with single point cane and modified independence. Conclusion This case reports describes the use of off-label EGT in addition to traditional physical therapy management that was successful in increasing the quality and efficiency of gait in an individual with polytrauma injuries and TBI+DAI.
\end{abstract}

Key words Exoskeleton gait training, Traumatic brain injury, Functional Independence

Corresponding Author Man-soo Ko(mako@utmb.edu)
Received date
19 January 2019
Revised date
01 February 2019
Accepted date
12 February 2019

\section{Introduction}

Individuals with diffuse axonal brain injury and polytrauma injuries present with a unique set of impairments and often lack awareness of their deficits. The Veterans Health Administration defines polytrauma as "two or more injuries, one of which may be life threatening, sustained in the same incident that affect multiple body parts or organ systems and result in physical, cognitive, psychological, or psychosocial impairments and functional disabilities." ") It also states that when brain injury is present it dictates the course of rehabilitation due to the complexity of cognitive, emotional, and behavioral deficits. ${ }^{1)}$

http:dx.doi.org/10.17817/2019.02.01.111368
Diffuse axonal injury (DAI) is a mechanism of traumatic brain injury (TBI) that causes excessive shearing of the white matter tracts over a widespread area. DAI is the predominant mechanism of injury in $40-50 \%$ of TBIs requiring hospital admission in the United States. ${ }^{2)}$ An element of DAI is theorized to be present in some capacity with all motor vehicle crashes causing the subject to lose consciousness. ${ }^{2)}$ It is believed that cognitive and motor deficits return at a decreased rate after a brain injury with DAI (TBI+DAI) as compared to brain injuries without DAI. This could be attributed to the effect that DAI has on the person's ability to learn new information and diminished capacity for information processing. ${ }^{2)}$

People with TBI present with a wide variety of activity and participation limitations. The spectrum of possible impairments include cognition, mobility, ac- 
tivities of daily living, and communication. This impacts the person's participation in family roles, social interactions, sustaining a job, and functioning as an active member of society. ${ }^{3)}$ For many persons with TBI, the main barrier to participation in a community setting is related to mobility. ${ }^{3)}$ The recovery of mobility is greatly impacted by commonly found impairments in strength, balance, coordination, voluntary motor control, and resurfacing primitive locomotion strategies. Improving walking function is often a vital component of the rehabilitation program to maximize the person's independence and help prevent the dependency on an assistive device or caregiver.

Rehabilitation techniques to utilize in persons with a diagnosis of TBI have historically been difficult to standardize in research because of the broad range of cognitive and motor deficits possible. Cognitive deficits in some specific areas, such as information processing and executive skills, seem to predominate. ${ }^{2}$ Kreutzer et al. ${ }^{4)}$ found that $87 \%$ of people with severe TBI had impaired performance on neuropsychological tests for rapid mental processing and $76 \%$ showed an impairment in memory for new information. ${ }^{4)}$ Clinically physical therapists must utilize treatment strategies to encourage information processing, retrieving and storing of new information to permit skill carry-over between treatment sessions.

Evidence is accumulating that repetitive, task specific practice, such as body weight support treadmill training (BWSTT) and exoskeleton gait training (EGT), should be used in the rehabilitation setting for people with TBI. ${ }^{5)}$ Repetition is thought to facilitate the integration of remaining and altered sensorimotor systems in persons with brain injury. ${ }^{3)}$ According to Schmidt et al. ${ }^{6}$ motor learning involves the integration of the sensory and motor information that occurs during practice. This becomes increasingly important when treating the TBI population because of the high likelihood of cognitive learning impairments impacting motor learning.

BWSTT is based on the current principle of motor learning through neuroplasticity. The repetition of normal physiological gait pattern with use of correct gait kinematics is hypothesized to target neuroplasticity. A recent study by Peters et al. ${ }^{7)}$ found that gait speed and mobility significantly improved in 10 subjects with brain injury after 20 sessions of BWSTT. Williams et al. ${ }^{8)}$ found that the manual assistance provided during BWSTT improved gait kinematics and the timing of muscle activation but was physically taxing on therapists. To replicate a normal gait pattern, historically 1-3 therapists are needed during BSWTT to control trunk and limb kinematics. A randomized comparative study by Esquenazi, et al. ${ }^{3)}$ compared robotic-assisted treadmill training (RATT) to manually assisted treadmill training (MATT). RATT-trained participants had significant improvement in step-length symmetry compared to MATT-trained participants and both groups had significant improvement in their overall mobility. RATT required fewer staff, less manual effort, and decreased staffing costs. ${ }^{3)}$ Consequently, robotic gait devices such as exoskeletons have emerged to assist in reducing this burden.

The benefits to EGT include a reduction in labor required from therapists, consistent movements, custom calculated gait kinematics, prolonged training times, and the possibility of over ground gait training unlike with BWSTT. Other evidence suggests that exoskeletons reduce immobility, improve spasticity, improve cardiopulmonary function, bowel and bladder automatic function, and have psychological benefits resulting in improved quality of life. $\left.{ }^{6}\right)$ Although a considerable amount of literature is available on the effectiveness of robotic gait devices in the spinal cord injury and stroke populations, there is little research regarding the TBI population.

The purpose of this case report was to describe the effects of EGT and traditional physical therapy management on physical performance, balance, and gait distance in a single individual with polytrauma injuries and TBI+DAI.

\section{Material and Methods}

\section{Patient History and clinical impression}

The subject was a 31 year old veteran male with an unremarkable past medical history. He was diagnosed 
with polytrauma injuries and TBI+DAI, resulting from a high speed motor vehicle collision. He was minimally responsive on the scene requiring immediate intubation by EMS. His injuries included TBI with DAI, ligamentum nuchae injury, right subarachnoid hemorrhage, right pneumothorax, ventilator-dependent respiratory failure, right maxillary sinus fracture, bilateral ear lacerations, left sternoclavicular joint dislocation, and left acromioclavicular joint dislocation. Initially he scored a 3 on the Glasgow Coma Scale for no spontaneous movement indicating severe brain injury. He began to emerge from a comatose state 8 days post-injury, was admitted to the Emerging Consciousness Program at an inpatient rehabilitation facility and was evaluated for the appropriateness of physical therapy 23 days post-injury. He reported his goals were to return to previous level of independence, remain employed with a labor-intensive job, and to "walk better and farther."

The subject did not have any medical complications that prohibited him from participating in therapy. Precautions included high fall risk and non-weight bearing to his left upper extremity. He presented to the evaluation with intermittent sharp left shoulder and neck pain that worsened with activity. The subject demonstrated ataxia of bilateral lower extremities and trunk with all movements. He was chosen for this case report due to the uniqueness of his medical diagnoses, his determination to return to his previous level to function independently as a father and employee, compliance with therapy recommendations, and limited amount of agitation or frustration making him a good candidate for EGT. Based on the subject's clinical presentation, it was decided to examine his ability to balance, transfer, and ambulate.

\section{Examination}

(1) Cognition

The subject was alert and oriented to person, place, date, and time. He presented with impairment of executive functioning, lack of deficit awareness, and mild impulsivity. He was easily redirectable to the task with frequent verbal cueing. He was able to follow simple one-step commands only and had diffi- culty sequencing multi-step tasks.

\section{Berg Balance Scale}

The participant performed the items of the Berg Balance Scale (BBS) as a measure of static and dynamic balance capability and to detect fall risk. Minimal detectable change and cut-off values are not determined for the TBI population, however in the elderly population meaningful change is indicated by an increase in score by 4.6 points and scoring less than $45 / 56$ indicates a high fall risk. The 14 -item balance test is recommended to be used with the TBI population in the inpatient rehabilitation setting by the TBI Taskforce." The subject was initially not appropriate to complete this outcome measure and was therefore scored as $0 / 56$ points.

\section{Functional Independence Measure}

The participant performed the segments of the Functional Independence Measure (FIM) as a measure of physical and cognitive function. The outcome measure was used to track the subject's level of disability and response to rehabilitation. Reported median correlation coefficients between total FIM scores are 0.95 for inter-rater reliability, 0.95 for test retest reliability, and 0.92 for equivalence reliability. ${ }^{10)}$ An admission FIM score of greater than 70 is associated with the patient achieving non-dependency at discharge. ${ }^{10)}$ The subject initially scored $62 / 126$ for the total FIM and $37 / 91$ on the motor subscale.

\section{(2) Gait Distance}

Gait distance was used as an outcome measure secondary to the patient's main goal to "walk better and farther" and the nature of his laboriously-intensive job requirements. To operationalize gait distance, the patient was provided the same verbal instructions each assessment. He was instructed to walk at his self-selected gait speed as far as he could before requiring a rest break. A second person was utilized to follow directly behind the subject with the same measuring wheel set to feet. The measurement would begin at the patient's feet when the therapist said, "go" and ended at the patient's feet when he took a seated or 
standing rest break. At initial evaluation the patient was able to ambulate 20 feet with a quad cane and moderate assistance from physical therapist.

\section{Disability Rating Scale}

The Disability Rating Scale (DRS) is intended for persons with moderate to severe TBIs and measures general recovery from coma to full recovery. It is designed to be used within 72 hours of rehabilitation admission and discharge with the maximum score of 29 indicating severe unresponsive wakefulness state. Testa et al. ${ }^{11)}$ found that the DRS was moderately predictive of long-term functional outcome, return to employment, and independent living. Initially the subject scored a 9/29 indicating he was markedly dependent for physical function and not employable.

\section{Intervention}

The subject participated in physical therapy one to two times daily for 60 minute sessions with five additional EGT sessions and two community re-integration outings, for an average of 7 sessions per week throughout the 5 week episode of care. Daily treatment sessions varied to include transfer, gait, balance, and strength training. Manual therapy to cervical and shoulder girdle musculature was used as needed for pain management. EGT was deemed appropriate and initiated 14 days after initial evaluation. Where progression for an activity was not described in the case study, the therapist added an appropriate exercise progression to maximize improvements. Activity performance was supervised by a physical therapist. The transfer training included repetitions of transfers from supine to sitting and sitting to standing. The subject initially required minimal assistance from the physical therapist with sit to stand transfers as well as constant verbal cueing for anterior weight shift and to prevent the subject from bracing his legs against the side of the mat. For this case, the goal was for the patient to eventually be able to perform these transfers with a single point cane at modified independence. The patient's body mechanics consistently improved with about ten repetitions per treatment session to encourage motor learning.
Over ground gait training (OGGT) was initiated at the first treatment session. Initial gait analysis discovered ataxia of bilateral lower extremities and trunk with narrow base of support and intermittent scissoring. He had inconsistent step length and cadence with difficulty sequencing the quad cane. EGT was initiated 14 days after initial evaluation as an attempt to reduce the dominant ataxic gait asymmetry. OGGT was utilized directly after EGT to encourage neuroplasticity. The subject participated in a total of five EGT sessions with the Ekso GT exoskeleton (Ekso Bionics, Richmond, CA). Appropriate fitting, donning of an abdominal binder, and the addition of padding to subject comfort was performed prior to powering on the device. This training required the assistance of two trained physical therapists for safe management of the patient in this device. The subject was able to follow commands appropriately for the safe use of the exoskeleton.

EGT utilized the ProStep Plus program allowing the subject to take consecutive steps in the robotic device. He was able to improve gait fluidity and maintain the desired forward and lateral trajectory targets with custom setting modifications. The subject spent about 60 minutes in the robotic device each EGT session with about 25 minutes of ambulation time. The remainder of the time was spent donning and doffing the device, making appropriate custom modifications as he progressed, and educating the subject during seated rest breaks. With each EGT session the subject demonstrated progress allowing for the decrease of the amount of assistance provided by the exoskeleton.

Balance training included a variety of combinations of eyes open, eye closed, firm ground, foam ground, rhomberg stance, sharpened rhomberg stance, and tandem stance. As the subject progressed, higher level dynamic balance tasks were added to the plan of care (e.g. resisted side stepping, mini-squats, stepping over obstacles). Dual task demands such as carrying objects, tossing balls, or counting backwards during ambulation were added in the fourth week once patient could tolerate the physical and cognitive demand.

Therapeutic exercise was used to increase the 
Table 2. Change in Gait Distance and Required Assistance by Week.

\begin{tabular}{cccccc} 
& Week 1 & Week 2 & Week 3 & Week 4 & Week 5 \\
\hline Gait Distance (feet) & 20 & 150 & 413 & 1887 & 5755 \\
Assistive Device & Quad Cane & Single Point Cane & Single Point Cane & Single Point Cane & Single Point Cane \\
Physical Assistance & Moderate & Minimum & Contact Guard & Supervision & Modified Independence \\
\hline
\end{tabular}

Table depicting the subject's maximal distance ambulated prior to requiring a rest break, the assistive device used, and the amount of assistance provided by the physical therapist. *Note the exponential gait distance increase during weeks 3,4 , and 5 after exoskeleton gait training was initiated at the end of week 2 .

strength of the participant's proximal hip musculature initially using blue theraband or two pound ankle cuff weights as resistance. Resistance was progressed as appropriate throughout plan of care. Exercises included supine bridges, straight leg raises, sidelying hip external rotation, sidelying hip abduction, and prone knee flexion. Heel raises, step-ups, squats, and lunges were added as the patient progressed.

He participated in two community re-integration outings during his fourth and fifth week of rehabilitation. One outing was to the grocery store where he was cognitively tasked to obtain the ingredients to make a meal within a certain budget and physically tasked to manage obstacles while scanning the environment. The second outing was a long community distance walk to a busy restaurant. He was tasked with safely navigating to and from the restaurant utilizing safe community ambulation skills.

\section{Results}

Data for outcome measures (BBS, FIM, gait distance) were obtained at admission to inpatient rehabilitation facility and were re-administered on a weekly basis
(Figure 1 and Table 1). The DRS was administered twice within 72 hours of rehabilitation admission and discharge as designed. The subject was able to progress with all treatment interventions targeting transfers, gait, balance, and proximal hip strength. He safely performed stand-pivot transfers and transfers from supine to sitting and sitting to standing with correct body mechanics 13 days after initial evaluation and reached modified independence with single point cane 25 days after initial evaluation.

There were immediate gait analysis improvements noted during over ground ambulation after each EGT session. The subject's step symmetry equalized, and his base of support widened with no episodes of scissoring gait pattern. The most noticeable change was the decrease in amount of ataxia present to bilateral lower extremities and trunk. With the reduction of gait deviations improving the energy efficiency of gait, the subject progressed from ambulating 20 feet with narrow-based quad cane and moderate assistance from the therapist at initial evaluation to ambulating 5,755 feet with single point cane at modified independence (Table 2).

The subject's BBS score increased from 0 to 54 . The FIM total score increased from 62 to 117 with the

\section{Table 1. Outcome Measure Scores by Week}

\begin{tabular}{cccccc} 
Measure & Week 1 & Week 2 & Week 3 & Week 4 & Week 5 \\
\hline Berg Balance Scale & 0 & 25 & 34 & 48 & 113 \\
Functional Independence Measure (total) & 62 & 93 & 102 & 717 \\
Functional Independence Measure (motor subscale) & 37 & 62 & 71 & 79 & 1887 \\
Gait Distance (feet) & 20 & 150 & 413 & - & 5755 \\
Disability Rating Scale & 9 & - & - & 2
\end{tabular}

Table depicting the subject's actual increase in outcome measure score at the beginning of each week during inpatient rehabilitation admission. Note week 1 values were from the initial physical therapy evaluation. Week 2 values were from the reassessment performed on the Monday of the subject's second week and so on. Week 5 values were obtained at the subject's discharge. Note the Disability Rating Scale was only performed at initial evaluation and discharge as intended. 
motor subscale increasing from 37 to 89 . The DRS score improved from 9 initially to 2 at discharge. The subject tolerated 5 weeks of physical therapy one to two times daily without any complications. The only negative impact was a slight increase in his sternoclavicular and left acromioclavicular joint pain during the first EGT session. The pain was eliminated with the addition of more padding above and below the left clavicle while in the exoskeleton.

\section{Discussion}

People with polytrauma injuries along with TBI+DAI present unique challenges to a physical therapist during comprehensive inpatient rehabilitation. The literature available to rehabilitation professionals regarding EGT in the TBI population is extremely limited. The purpose of this case report was to describe the use of EGT and traditional physical therapy management of an adult with polytrauma injuries and TBI+DAI during an inpatient rehabilitation stay. Key findings can be summarized as the subject made marked improvements with all outcome measures and the utilization of EGT followed by OGGT had immediate reduction in dominant ataxic gait deviations. The reduction of gait deviations decreased the energy burden of gait allowing the subject to increase his walking endurance and decrease the dependence on an assistive device and caregiver.

The use of the Ekso GT exoskeleton for this subject with the diagnosis of TBI was an off-label use. The FDA has approved the use of the Ekso GT for patient populations including individuals with hemiplegia due to stroke, spinal cord injuries T4 to L5, and spinal cord injuries at levels $\mathrm{C} 7$ to T3 ASIA D. ${ }^{12)}$ However, the Certification Marking Indications for Use states the device is intended for any patient with a neurological or muscular injury, illness, or weakness. ${ }^{12)}$ Ekso GT trained physical therapists made the clinical decision to train this subject in the exoskeleton off-label because of his high cognitive function and limited amount of agitation or frustration in therapy sessions. It was believed that the patient

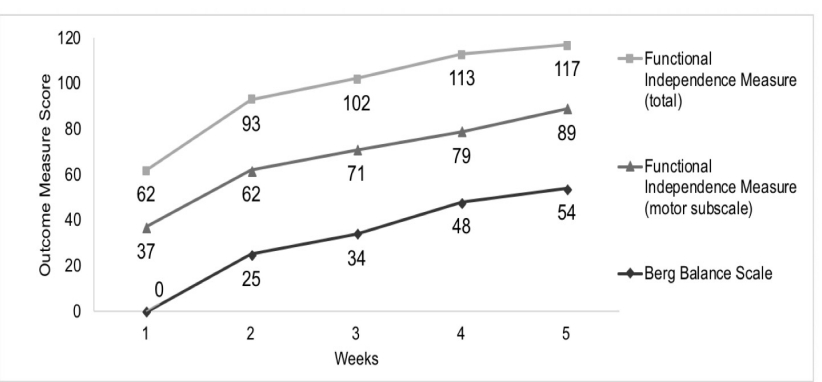

Figure 1. Outcome Measure Totals by Week.

Graph depicting the subject's actual increase in outcome measure score at the beginning of each week during his stay in an inpatient rehabilitation facility. Note that week 1 values are from the initial physical therapy evaluation. Week 2 values were from the reassessment performed on the Monday of the subject's second week and so on. Week 5 values were obtained at the subject's discharge.

would tolerate feedback constructively, apply the education to OGGT, and remain safe while wearing the exoskeleton. Prior to the initiation of EGT the subject showed limited improvements in his bilateral lower extremity and truncal ataxic gait deviations. When combined with traditional physical therapy management including balance and strength training, the plan of care was successful in increasing the quality and efficiency of gait.

Rehabilitation techniques for persons with TBI have historically been difficult to research because of the broad range of cognitive and motor deficits. Repetitive, task specific practice, such as BWSTT or EGT, could prove viable for people with TBI because repetition is thought to facilitate the integration of remaining and altered sensorimotor systems. ${ }^{3)}$ In this case, the subject showed limited reduction in his gait deviations prior to the initiation of EGT including ataxic movements of bilateral lower extremities and trunk, narrow base of support, intermittent scissoring, inconsistent step length, and variable cadence. After the fourth EGT session the subject stated, "It helps me, kind of like muscle memory. It walks how I should walk so when I get out of it, my body knows that it needs to walk that certain way." The subject completed five EGT sessions until the subject demonstrated ambulation recovery with minimal gait deviations and was thought to benefit from higher-level balance and gait training. Ultimately his BBS score in- 
creased from 0 to 54 and DRS improved from 9 to 2. While this case study provides important information for clinicians, there are limitations to be considered. First, this is a case study of a single subject and it is unclear whether these results are generalizable to the larger population of people with TBI+DAI as there are a broad range of cognitive and motor deficits possible. Gait distance was used as an outcome measure with the rational that diminishing gait deviations will reduce the energy burden of gait thusly increasing the gait distance the subject could ambulate prior to requiring a rest break. However, this case study would have benefitted from the use of a more objective outcome measure recommended for the TBI population such as the 6 minute walk test.

In conclusion, this case report provides an example of incorporating EGT into a traditional physical therapy plan of care that was successfully implemented in a veteran with polytrauma injuries along with TBI+DAI. He tolerated 5 weeks of physical therapy one to two times daily without any complications during the entirety of his inpatient rehabilitation stay. He had significant score increase with the BBS, FIM total, FIM motor subscale, and DRS. More work is needed to determine the appropriateness of the use of an exoskeleton for gait training in the TBI population, and if appropriate then treatment guidelines to maximize functional neuroplastic return.

\section{References}

1. Petzel RA. Polytrauma System of Care. Department of Veterans Affairs, Veterans Health Administration Directive. 2013; p. 2.

2. Meythaler JM, Peduzzi JD, Eleftheriou E, Novack TA. Current concepts: diffuse axonal injury-associated traumatic brain injury. Arch Phys Med Rehabil. 2001;82:1461-1471.

3. Esquenazi A, Lee S, Packel AT, Braitman L. A randomized comparative student of manually assisted verus robotis-assisted body weight supported treadmill training in persons with a traumatic brain injury. American Academy of Physical Medicine and Rehabilitation. 2013;5:280-290.
4. Kreutzer JS, Gordon WA, Rosenthal M, Marwitz J. Neuropsychological characteristics of patients with brain injury: preliminary findings from a multicenter investigation. Journal of Head Trauma Rehabilitation. 1993;8(2):47-59.

5. Esquenazi A, Lee S, Wikoff A, Packel A, Toczylowski T, Feeley J. A comparison of locomotor therapy interventions: partial-body weight-supported treadmill, lokomat, and G-EO training in people with traumatic brain injury. American Academy of Physical Medicine and Rehabilitation. 2017;9:839-846.

6. Schmidt R, Lee T, Winstein C, Wulf G, Zelaznik H. Motor control and Learning: A behavioral Emphasis. Champaign, IL: Human Kinetic Publishers: 1998.

7. Peters DM, Jain S, Liuzzo DM, Middleton A, Greene J, Blanck E, Sun S, Raman R, Fritz SL. Individuals with chronic traumatic brain injury improve walking speed and mobility with intensity mobility training. Archives of Physical Medicine and Rehabilitation. 2014; 95:1454-60.

8. Williams G, Clark R, Schache A, Fini NA, Moore L, Morris ME, McCrory PR. Training conditions influence walking kinematics and self-selected walking speed in patients with neurological impairments. Journal of Neurotrauma. 2011;28:281-287.

9. McCulloch K, De Joya A, Donnelly E, Hays K, Johnson TK, Nirider C, Roth H, Saliga S, Ward I. TBIEDGE outcome measures for inpatient and outpatient rehabilitation. Academy of Neurologic Physical Therapy. http://www.neuropt.org/docs/tbi-edge/tbi-edge-neuro-se ction-full-edge-documents.pdf. February 2012. Accessed March 24, 2018.

10. Mackintosh S. Functional Independence Measure. Australian Journal of Physiotherapy. 2009;55:65.

11. Testa JA, Malec JF, Moessner AM, Brown AW. Outcome after traumatic brain injury: effects of aging on recovery. Archives of Physical Medicine and Rehabilitation. 2005;86:1815-23.

12. Supporting Better Patient Outcomes. Ekso Bionics. https://eksobionics.com/eksohealth/patients. Accessed March 24, 2018. 
